\title{
Saved by the bell: Early surgical treatment for cardiosystemic thromboembolism
}

\author{
Dumbor L. Ngaage, FRCS, Gurpreet S. Bhamra, MRCS, Sydney Gaynor, FRCS, and Unnikrishna R. Nair, FRCS, \\ Leeds, United Kingdom
}

$\mathrm{T}$ he combination of pulmonary embolism (PE), paradoxical embolism (PDE), and an intracardiac thromboembolus trapped in a patent foramen ovale (PFO) can be described as cardiosystemic thromboembolism. PE is associated with an increase in the right heart pressures precipitating a right-to-left shunt through a PFO and consequently predisposing to PDE. This probably accounts for more cases of arterial embolism encountered in clinical practice than is realized. The right atrial thromboembolus can lodge in the PFO (impending PDE) and become a focus of further growth and systemic embolization. This warrants prompt and expeditious elimination of the thromboembolus, but unfortunately opinions still vary on the best management strategy.

\section{Clinical Summary}

A 72-year-old white woman had a right axillary embolism 3 weeks after a total right knee replacement for primary osteoarthritis. An emergency embolectomy was then performed and a $30 \times 15-\mathrm{mm}$ embolus was removed. Five days later, sudden-onset dyspnea and chest pain developed.

She had undergone bilateral varicose vein stripping and hemorrhoidectomy in the past and was being treated for hypertension and chronic stable angina.

On admission, she was sweaty and tachypneic at 36 breaths/ min with an oxygen saturation of $85 \%$ while receiving $4 \mathrm{~L}$ of oxygen by face mask. She had a heart rate of 90 beats $/ \mathrm{min}$, a blood pressure of 160/80 $\mathrm{mm} \mathrm{Hg}$, and normal jugular venous pressure. Arterial blood gas analysis showed a $\mathrm{Po}_{2}$ of $6.1 \mathrm{kPa}$, a $\mathrm{PCO}_{2}$ of 4.1 $\mathrm{kPa}$, and a $\mathrm{pH}$ of 7.47. There was a 2-mm ST elevation in leads III and aVF of the electrocardiogram. Hematology, biochemistry, and cardiac enzyme levels were normal. The chest radiograph showed no abnormalities, but computed tomographic and ventilation-perfusion scans revealed a small pulmonary embolism affecting the right upper lobe. Transesophageal echocardiography demonstrated a dumbbell-shaped thromboembolus lodged in a PFO (Figure 1).

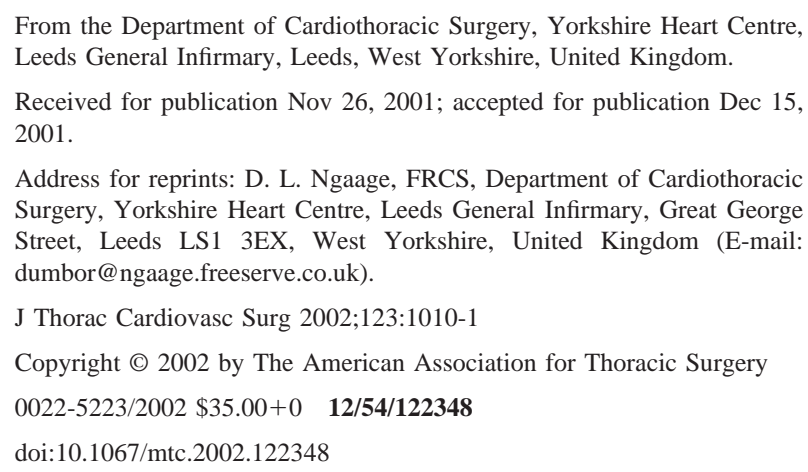

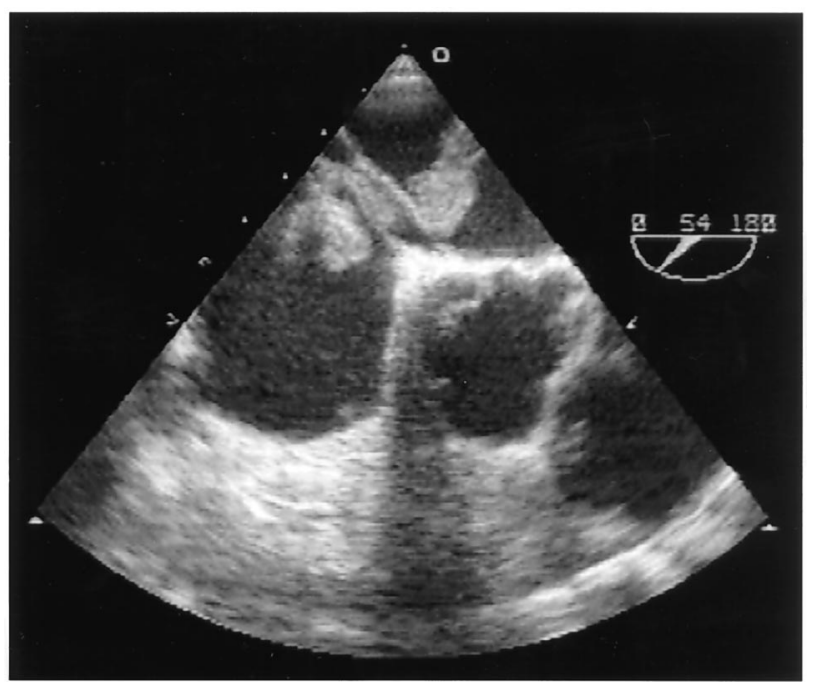

Figure 1. A 4-chamber view of the transesophageal echocardiogram showing a large saddle thromboembolus in both the left and right atria. The thromboembolus extends through a PFO from the right atrium to the left atrium.

An emergency cardiac thromboembolectomy was performed through a median sternotomy with the aid of cardiopulmonary bypass (Figure 2). The thrombus was retrieved through a right atriotomy and the PFO closed directly. The left heart chambers were explored through a left atriotomy posterior and parallel to the interatrial groove.

The patient made an uneventful recovery and was discharged home receiving warfarin on the seventh postoperative day.

\section{Discussion}

A PFO is a potential channel for the arterial embolization of an intracardiac thromboembolus. Anatomic persistence of the foramen ovale, a feature of fetal circulation, occurs in $25.4 \%$ of persons between 30 and 79 years of age. ${ }^{1}$ It is usually not associated with physiologic activity, but interatrial shunting occurs when an alteration in the hemodynamics engenders a rise in right atrial pressures. A functional PFO should be suspected in a patient who has an arterial embolic event and a history of venous thrombosis, ${ }^{2}$ as this case clearly illustrates. In 1972, Meister and associates ${ }^{3}$ proposed that the combination of arterial embolism, venous thrombosis, intracardiac defect, and a transient reversal of the intracardiac shunt were required for the diagnosis of PDE. With the widespread use of echocardiography, it has become a vital diagnostic tool in this condition. The demonstration of an intracardiac thromboembolus within the PFO on echocardiography, which is 


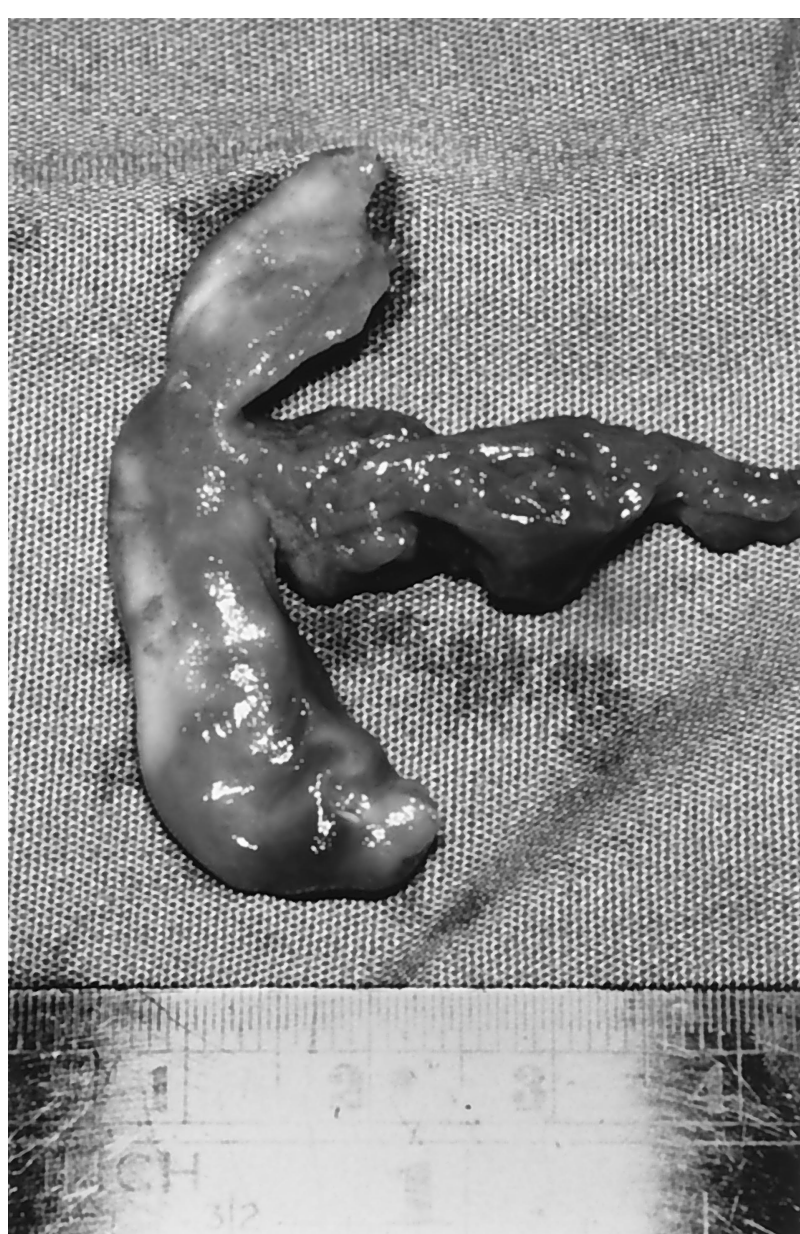

Figure 2. Photograph of the thromboembolus after surgical removal.

rare in clinical practice, in association with arterial embolism is diagnostic of PDE.

The thromboembolus trapped in the PFO is in an unstable pathophysiologic state because the perturbations of the constantly changing intracardiac pressures subject it to varying shearing forces. As a result, it tends to fragment and cause recurrent PE and
PDE. Impending PDE is therefore a transitional state presaging a catastrophic embolic event. In our patient, the dumbbell shape of the impending PDE probably provided a temporary stabilizing effect and prevented a rapid progression to massive PDE. Hence it is of utmost importance to remove the entrapped thromboembolus expeditiously, especially after a "herald" arterial embolism as in this patient.

Treatment options for this condition are still varied. In 1998, Aboyans and associates ${ }^{4}$ reviewed the treatment modalities of 39 reported cases of PDE and found that surgery has the lowest mortality ( $15.8 \%$ vs $57 \%$ for anticoagulation alone vs $20 \%$ for thrombolysis). They also noted that persons treated by surgery, rather than the other treatment methods, did not have recurrent embolism. In our experience, prompt operative treatment for cardiosystemic thromboembolism is safe, enables a direct visualization of the left heart chambers and pulmonary tree, and also allows a complete embolectomy with the help of cardiopulmonary endoscopy. ${ }^{5}$ This was not necessary in this patient because she did not have significant PE. Surgical treatment also allows a permanent closure of the PFO and prevents recurrent PDE. ${ }^{6}$

In conclusion, prompt operative treatment of impending PDE provides an immediate elimination of the potentially catastrophic consequences of cardiosystemic embolism.

\section{References}

1. Hagen PT, Scholz DG, Edwards WD. Incidence and size of patent foramen ovale during the 10 decades of life: an autopsy study of 956 normal hearts. Mayo Clin Proc. 1984;59:17-20.

2. d'audiffret A, Pillai L, Dryjski M. Paradoxical emboli: the relationship between patent foramen ovale, deep vein thrombosis and ischaemic stroke. Eur J Vasc Endovasc Surg. 1999;17:468-71.

3. Meister SG, Grossman W, Dexter L, Dalen JE. Paradoxical embolism. Am J Med. 1972;53:292-8.

4. Aboyans V, Lacroix P, Ostyn E, Cornu E, Laskar M. Diagnosis and management of entrapped embolus through a patent foramen ovale. Eur J Cardiothorac Surg. 1998;14:624-8.

5. Ngaage DL, Shah R, Sanjay SP, Cale ARJ. Cardiopulmonary endoscopy: an effective and low risk method of examining the cardiopulmonary system during cardiac surgery. Eur J Cardiothorac Surg. 2001;19:152-5.

6. Dearani JA, Ugurlu BS, Danielson GK, Daly RC, McGregor CG, Mullany CJ, et al. Surgical patent foramen ovale closure for prevention of paradoxical embolism-related cerebrovascular ischemic events. Circulation. 1999;100(19 Suppl):II-171-5 . 